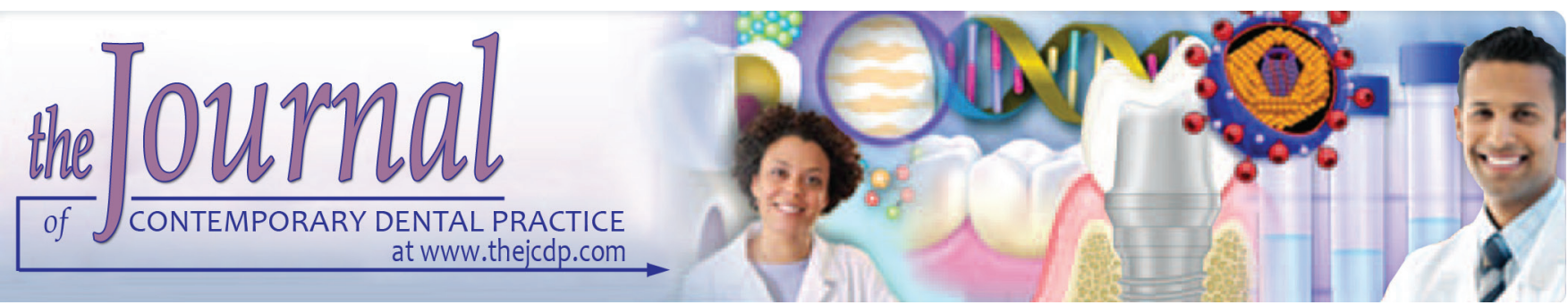

\title{
Higher Dental Caries Prevalence and Its Association with Dietary Habits and Physical Limitation in Epidermolysis Bullosa Patients: A Case Control Study
}

\footnotetext{
${ }^{1}$ Soraya Coelho Leal, ${ }^{2}$ Erica Negrini Lia, ${ }^{3}$ Rivadavio Amorim, ${ }^{4}$ Mateus Veppo dos Santos, ${ }^{5}$ Mayara Cundari de Araújo
}

${ }^{6}$ Rodrigo Antônio de Medeiros, ${ }^{7}$ Camila Abdanur da Fonseca

\begin{abstract}
Aim: Epidermolysis bullosa (EB) represents a highly rare subgroup of skin diseases that affects skin and mucous membrane. The aim of the present study was to assess caries prevalence and its associated factors in EB subjects. Salivary status was also assessed.

Materials and methods: Ten subjects with EB who were under supervision were selected (cases) and matched by age and gender with unaffected individuals (controls). Dental caries were recorded using the World Health Organization (WHO) criteria. Oral hygiene and dietary habits were investigated by clinical examination and questionnaires. Both nonstimulated and stimulated saliva were collected and salivary $\mathrm{pH}$, buffering capacity and mouth opening were evaluated.
\end{abstract}

Results: The results showed that the median decay-missingfilled teeth was significantly higher $(p=0.0094)$ in EB cases 5 (3.9-20.3) than in controls 3 (2-3.25). The groups also differed when food consistency was analyzed. Individuals with EB have a higher intake of soft food. In addition, the median mouth-opening values from cases $(0.84-2.84 \mathrm{~cm})$ and controls $(4.3-4.9 \mathrm{~cm})$ have shown to be statistically different $(p=0.007)$. Considering

\footnotetext{
1,2,4,5,7 Department of Dentistry, School of Health Sciences University of Brasília (UnB), Brasília-DF, Brazil

${ }^{3}$ Department of Physical Medicine and Rehabilitation Spaulding Rehabilitation Hospital, Harvard Medical School Boston, Massachusetts, USA

${ }^{6}$ Department of Dental Materials and Prosthodontic, School of Dentistry, São Paulo State University (UNESP), São Paulo, Brazil

Corresponding Author: Erica Negrini Lia, Professor Faculdade de Ciências da Saúde, Departamento de Odontologia, Universidade de Brasília (UnB) Campus Darcy Ribeiro, Brasília-DF 70910-900, Brazil, Phone: +556131071803 e-mail: ericalia@unb.br
}

the salivary parameters, none of them showed significant differences among groups.

Conclusion: Epidermolysis bullosa subjects present higher caries scores and might be related to their physical condition and dietary habits.

Clinical significance: There is a lack of information about oral status in EB subjects. Hence, our findings add useful information regarding the relationship between caries prevalence and associated risk factors in EB subjects.

Keywords: Caries, Case control, Epidermolysis bullosa, Oral health, Saliva.

How to cite this article: Leal SC, Lia EN, Amorim R, dos Santos MV, de Araújo MC, de Medeiros RA, da Fonseca CA. Higher Dental Caries Prevalence and its Association with Dietary Habits and Physical Limitation in Epidermolysis Bullosa Patients: A Case Control Study. J Contemp Dent Pract 2016;17(3):211-216.

Source of support: Nil

Conflicts of interest: The authors followed the International Committee or Journal of Medical Journals Editors (ICMJE) form for disclosure of potential conflicts of interest. All listed authors concur with the submission of the manuscript; all authors have approved the final version. The authors have no financial or personal conflicts of interest.

\section{INTRODUCTION}

The genodermatoses are a vast and heterogeneous group of cutaneous diseases whose etiology is associated with defects and/or genetic alterations. Among these diseases stands out the epidermolysis bullosa (EB) that represents a subgroup of skin diseases related to extracutaneous alterations. It is characterized by blisters and/or erosions in the skin and mucous membrane either after mechanical trauma or spontaneously. ${ }^{1,2}$

A revised classification system divided the inherited EB in four major types: epidermolysis bullosa simplex 
(EBS), junctional epidermolysis bullosa (JEB), dystrophic epidermolysis bullosa (DEB), and Kindler syndrome, based on distinguishing ultrastructural sites of blister formation. $^{3}$

Epidermolysis bullosa is a complex and an incurable disease that affects people throughout the world. Its precise prevalence is not known, but studies conducted in different parts of the world show that it can vary from 1 to 49 per one million live births. ${ }^{4,5}$ Although it is considered rare, it has devastating effects on patient's quality of life. ${ }^{5}$ Both extracutaneous complications and the prognosis of EB are highly dependent on the subtype of disease that is present. In general, patients suffering from EBS and DEB types have normal life expectancies, while those who present the JEB form are at a high risk of death during the first years of life. ${ }^{5}$

Oral manifestations related to EB are usually observed, but can vary tremendously in both character and severity according to the EB type. ${ }^{6}$ For example, enamel hypoplasia is seen in all subtypes of $\mathrm{JEB},{ }^{7}$ while in patients with recessive $\mathrm{DEB}$, severe ankyloglossia and microstomia are usually observed. ${ }^{5}$ Repeated blistering and scar formation at soft tissues in perioral cavity lead to limited mouth opening, elimination of buccal and vestibular sulci. ${ }^{6,7}$ In addition, restrictions in mobility of the mouth, tongue, and lips are frequently present in EB patients. ${ }^{8}$ In terms of dental caries, there are just few published papers addressing the issue that patients with EB are at a high risk of developing the disease. 6,9,10 It has been proposed that the increased fragility of oral soft tissues in patients with EB may lead to changes in diet and difficulties in properly performing oral hygiene, factors that can be related to the increased risk of caries of these patients. ${ }^{6,11}$ However, other factors such as saliva $\mathrm{pH}$ and buffering capacity, which are known to be associated with dental caries, ${ }^{12}$ have not been yet extensively investigated in patients with EB. Therefore, considering that there is a lack of information about dental caries and variables associated with its development in patients with $\mathrm{EB}$, the present investigation aimed to compare the caries prevalence and to investigate factors that could be associated to caries development in patients with EB in comparison to those who do not have the disease.

\section{MATERIALS AND METHODS}

\section{Study Population}

The present descriptive case-control study was conducted at the Paediatric Dentistry Clinics at the University Hospital and was approved by Institutional Review Board (IRB). Cases were selected according to the following criteria: To present EB of any type and to be under medical supervision at the Dermatology Service from the same hospital. Cases and controls were matched in relation to age, gender, and socialeconomic status. The experiments were undertaken with the understanding and written consent of each subject and according to Declaration of Helsinki.

\section{Clinical Evaluation}

Participants were submitted to a set of procedures in two different moments. During the first visit, the sequence of procedures were an interview wherein information regarding oral health habits, including the frequency of daily tooth brushing, toothbrush size, the use of dental floss, and type and amount of toothpaste, was performed; a 24-hour recall dietary form was filled in with information about the participants diet habits - number of daily meals and the menu description, including food consistency; an oral examination was conducted under ideal lightening conditions, to record the number of decayed, missed and filled teeth, using the WHO criterion by one calibrated examiner ${ }^{13}$; and maximum mouth opening was registered using a pachymeter that was positioned from the superior incisor to the inferior one, having the medium line as a reference point (Fig. 1).

\section{Salivary Analysis}

At the second visit, saliva assessment was performed considering the following sequence: Mucosa hydration test was performed by everting the lower lip and gently drying the mucosa with a small piece of gauze. With the assistance of a chronometer, time taken to observe droplets of saliva forming at the orifices of the minor salivary glands was recorded. It was considered that if the time needed was superior to 60 seconds, the resting flow rate is below normal ${ }^{14}$; and nonstimulated and stimulated saliva were collected during 3 minutes each. Individuals were instructed not to eat, drink, or smoke 1 hour

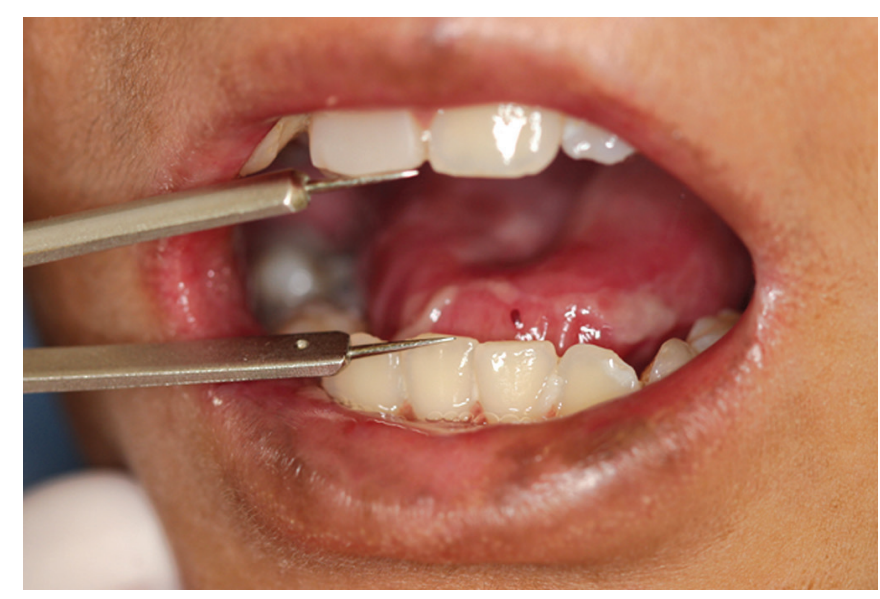

Fig. 1: Procedure used for the measurement of mouth opening 
before the examination, which was performed between 9.00 and 11:00 am to minimize variations associated with the circadian cycle. Initially, nonstimulated saliva was collected into grade glass container previously cooled to avoid foam formation. Stimulated saliva was then collected in another grade glass, while participants were chewing a paraffin wax. ${ }^{15}$ The $\mathrm{pH}$ was analyzed using a digital pH measurer (MPH 100-Minpa, São Paulo, Brazil) from $2 \mathrm{ml}$ of stimulated saliva. Immediately after, the buffering capacity analysis was performed by adding $0.5 \mathrm{ml}$ of $\mathrm{HCl}$ $(0.05 \mathrm{~N})$ volume to the saliva sample and registering the final value. This procedure was repeated three times, with a final acid volume of $2 \mathrm{ml}$. To calculate the buffering capacity, the Van Slyke formula was used: $\beta=\Delta \mathrm{Ca} / \Delta \mathrm{pH}$, where $\beta$ is the buffering capacity, $\Delta \mathrm{Ca}$ is the quantity of acid added in grams per liter, and $\Delta \mathrm{pH}$ is the change in $\mathrm{pH}$ induced by the acid addition. ${ }^{16}$

\section{Statistical Analysis}

Statistical analysis was performed using the software GraphPad Prism-6.00 for the Windows GraphPad Software, La Jolla, California. D’Agostino-Pearson omnibus test was used to assess data normality. For the variables with normal distribution, $t$ tests were applied using Welch correction for those cases in which the standard deviation differed between the groups. Mann-Whitney test was used for nonparametric analysis. Spearman correlation test was used for age and decaymissing-filled teeth (DMFT) scores.

\section{RESULTS}

\section{Sample Characteristics}

A total of 20 individuals with a mean age of $18.2( \pm 13.8)$ years were divided in cases $(n=10)$ with EB and in controls $(n=10)$, without $E B$, matched by age and gender. The majority of individuals was female (eight per group). Half of the sample was comprised by children (6-10 years old), one adolescent (15 years old), and four adults (30-48 years old).

All individuals with EB included in this study, except one, presented the recessive dystrophic EB type. The other subject did not have a confirmatory examination for the EB type, but due to the clinical symptoms of the disease, which was less severe in this patient, there was speculated that he presented the simplex form of EB.

\section{Oral Hygiene and Dietary Habits}

It was observed that tooth-brushing frequency was similar between the two groups and that individuals without EB used the dental floss more regularly in comparison to the ones with EB. The type of toothbrush differed between groups, as even the adults with EB preferred to use the infant toothbrush. They also preferred to use the infant toothpaste (Table 1).

Regarding diet, it was observed that individuals from both groups reported to eat, in average, five times a day with a similar ingestion of sugar content. However, the groups differed when food consistency were analyzed. Individuals with EB informed that they preferred soft food. In addition, all individuals with EB reported the consumption of a nutritional supplement, with a high concentration of sucrose, twice a day as part of their regular diet.

\section{Caries Prevalence}

The median DMFT scores of cases and controls were 5 (3.9-20.3) and $3(2-3.25)$ respectively and differed statistically $(\mathrm{p}=0.0094)$. The highest DMFT score found for patients with EB was 28 (Figs 2A and B) and for those

Table 1: Oral hygiene frequency, type of toothpaste, and toothbrush preference

\begin{tabular}{|c|c|c|c|c|}
\hline & Cases & & Controls & \\
\hline $\begin{array}{l}\text { Tooth- } \\
\text { brushing } \\
\text { frequency }\end{array}$ & 2.7 times/day & & 3 times/day & \\
\hline $\begin{array}{l}\text { Dental } \\
\text { floss use }\end{array}$ & Yes $=20 \%$ & $\mathrm{No}=80 \%$ & Yes $=40 \%$ & $\mathrm{No}=60 \%$ \\
\hline $\begin{array}{l}\text { Toothbrush } \\
\text { type }\end{array}$ & Infant = 100\% & Adult $=0 \%$ & Infant $=50 \%$ & Adult $=50 \%$ \\
\hline $\begin{array}{l}\text { Toothpaste } \\
\text { type }\end{array}$ & Infant $=60 \%$ & Adult $=40 \%$ & Infant $=30 \%$ & Adult $=70 \%$ \\
\hline
\end{tabular}
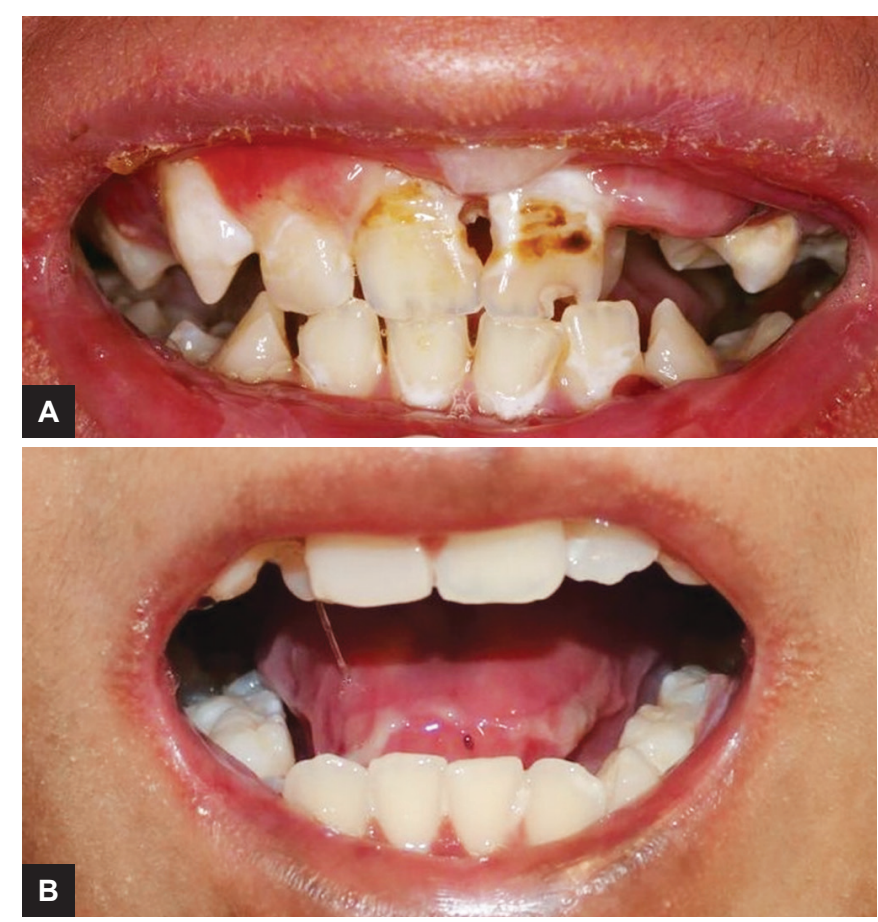

Figs 2A and B: (A) Cavitated dentine lesions present in an EB subject (top) and (B) Visible dental plaque and tongue ulcers (bottom) 
without EB was 14. The correlation between median caries scores and age is presented in Graph 1.

\section{Saliva and Mouth Opening}

None of the saliva aspects investigated - mucosa hydration, salivary flow, $\mathrm{pH}$, and buffer capacity differed statistically between the groups (Tables 2 and 3). The median of the mouth opening, in centimeters, differed statistically significant between cases and controls. Individuals with EB showed values varying from 0.84 to $2.84 \mathrm{~cm}$, while the ones without EB presented higher values that varied from 4.3 to $4.9 \mathrm{~cm}(\mathrm{p}=0.0007)$.

\section{DISCUSSION}

In general, the literature shows that individuals with EB are at a high risk for caries development. ${ }^{6,10}$ Epithelial fragility that leads to oral mucosa blistering, varying in severity according to the EB subtype, is associated with difficulties in proper teeth cleaning. ${ }^{6,8}$ The dietary pattern of individuals with EB is also influenced by the disease itself, contributing to the impaired oral intake of solid food. ${ }^{6,7}$ However, in which extent these oral manifestations of EB are associated with the development of dental caries? It is well known that saliva plays an important role in maintaining oral balance and that salivary flow is stimulated by mastication. Therefore, it is of interest to learn whether salivary characteristics are also influenced by the disease itself and/or by the oral health conditions of these individuals, once the mastication pattern is altered in individuals with EB.

In order to discuss in detail the oral health findings of our individuals, it is important to highlight that all, except one subject, presented with the most severe EB subtypeDEB. This aspect should be taken into account, as the EB type influences the severity of the oral manifestations of the disease. Another aspect that also needs to be considered is the fact that the adolescent and the adults patients with EB who comprised our sample were being followed-up by the hospital multicenter health team for a long period. In such condition, both patients and/or caregiver have been constantly instructed about diet and oral hygiene habits; therefore, one might infer that the oral health status of our population should be in a better condition than others patients with EB, but that are not regularly seen by a dentist.

The outcomes regarding oral hygiene showed that both cases and controls presented similar tooth brushing frequency, but due to the fragility of their mucosa, the individuals with EB preferred infant toothbrushes, that
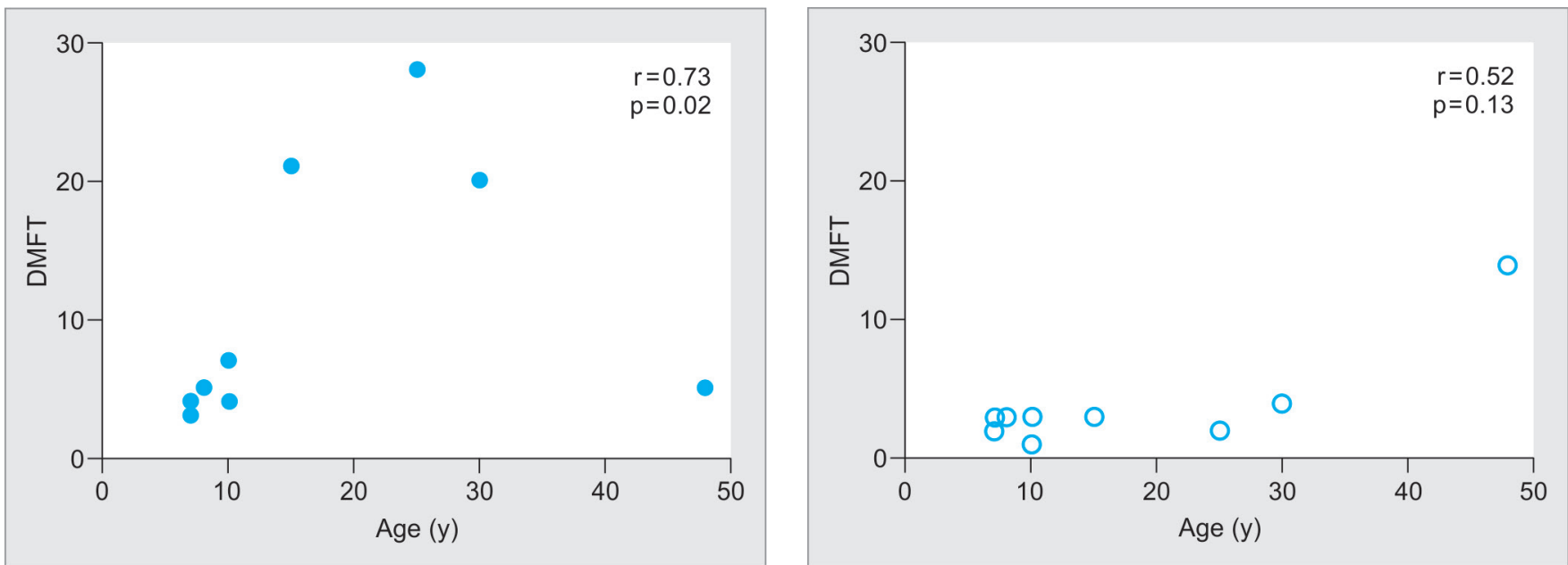

Graph 1: Correlation between DMFT and age for cases and controls (respectively) is shown in the right and left graphs respectively. "r" $\mathrm{s}$ Spearman $\mathrm{r}$ correlation value

Table 2: Mucosa hydration time, salivary stimulated, and unstimulated flow rates considering cases and controls

\begin{tabular}{llll}
\hline & $\begin{array}{l}\text { Mucosa } \\
\text { hydration }^{*}(\mathrm{sec})\end{array}$ & $\begin{array}{l}\text { Stimulated } \\
\text { saliva** } \\
(\mathrm{mL} / \mathrm{min})\end{array}$ & $\begin{array}{l}\text { Unstimulated } \\
\text { saliva** } \\
(\mathrm{mL} / \mathrm{min})\end{array}$ \\
\hline Cases & $11.6( \pm 2.63)$ & $0.9(0.6-1.1)$ & $1.2(0.8-2.6)$ \\
Controls & $10.5( \pm 2.79)$ & $1.0(0.8-1.3)$ & $1.8(1.2-1.9)$ \\
\hline p-value & 0.32 & 0.32 & 0.34 \\
\hline
\end{tabular}

*Values expressed by mean and standard deviation; **Values expresses by median and interquartile ranges (IQRs)
Table 3: $\mathrm{pH}$ and buffer capacity of individuals with and without EB

\begin{tabular}{cclll}
\hline \multicolumn{2}{c}{$p H$ (mean and SD) } & & \multicolumn{2}{l}{ Buffer capacity (median and IQR) } \\
\cline { 1 - 1 } Cases & Controls & & Cases & Controls \\
\cline { 1 - 1 } $7.0(7.0-7.2)$ & $7.1(7.0-7.2)$ & & 0.003 & 0.005 \\
& & & $(0.002-0.007)$ & $(0.002-0.01)$ \\
\hline & $p=0.27$ & & $p=0.27$ \\
\hline
\end{tabular}


in general, are smaller and have softer bristles. The frequency of the dental floss use was also lower in EB patients as in most cases; they were not able to do it by themselves as a consequence of their hands/finger atrophy and also due to the mucosa fragility. Difficulties in proper gripping and holding a toothbrush due to the hands and fingers blistering, ${ }^{6}$ as well as pain caused by ulcerations of oral mucosa ${ }^{8}$ have already been considered as barriers to oral hygiene in individuals with EB.

Ideally, it would have been interesting registering both visible plaque (VPI) and gingival bleeding (GBI) indexes prior to the oral examination. However, in the present study, most of the EB individuals could not properly open their mouth to allow the examination of the buccal surfaces of the posterior teeth and bleeding not associated with dental plaque might occur in individuals with EB. Therefore, to avoid introducing bias during the clinical examination, the decision to not record VPI and GBI was made. However, it can be speculated that, due to the difficulties in brushing the teeth reported by the individuals with EB, more plaque and gingival bleeding would be a natural finding in this population. According to Harris et al, ${ }^{6}$ a greater mean plaque score and mean gingivitis score were observed in patients with dystrophic EB than in nondiseased subjects.

Regarding diet pattern analysis, it was observed that the frequency and sugar content did not differ between cases and controls. However, the food consistency of those with EB was predominantly soft. The mastication of hard and/or fibrotic food might cause trauma to the soft tissues; blisters can be formed immediately. Therefore, in order to avoid discomfort during eating, individuals with EB have a preference for soft food., 5 It is well known that the importance of the mechanical stimulus for producing stimulated saliva and that the composition of stimulated and nonstimulated saliva differs considerably. ${ }^{12,17}$ For that reason, it is extremely relevant to investigate whether the pattern of food intake of individuals would interfere on saliva properties, such as $\mathrm{pH}$ and buffer capacity.

Although a direct relationship between saliva characteristics and dental caries has not been established for individuals with a normal saliva production, there are studies that show that in adverse conditions, such as salivary gland atrophy, the reduced amount and/ or lack of saliva is associated with the presence of rampant caries. ${ }^{18}$ Our results showed that even though the values for all salivary parameters (saliva flow rate, $\mathrm{pH}$, and buffer capacity) obtained from individuals with EB were slightly lower than for those without EB, there were no significant differences between cases and controls. Similar results were observed by a study of Wright et al, $^{19}$ in which individuals with EB did not show a decreased saliva flow in comparison to unaffected subjects. However, in relation to $\mathrm{pH}$ and buffer capacity, the present investigation is the first one that suggests that they are also not influenced by EB. As a consequence, the differences on caries prevalence observed between EB and none EB persons could not be related to saliva flow rate, $\mathrm{pH}$, and buffer capacity and reinforces the concept that caries pattern in severely affected EB individuals is likely attributable to nonsalivary factors. ${ }^{9}$

Additionally, all EB individuals used to ingest a nutritional supplement containing a high sucrose content that is taken in a creamy form twice a day. However, the supplement ingestion was usually performed after the main meals, being immediately followed by a tooth brushing. Therefore, the sugary supplement consumption could not also be associated with caries prevalence. This is valid for this population and might not be extrapolated to all and every EB individual, as the ones that were investigated herein are continuously advised during their routine dental visits about the importance of oral health cleaning after meals. Nevertheless, the advice and the follow-up visits were not enough to avoid the development of dental caries. In the present study, caries prevalence increased in both cases and controls with age, but caries increment was much severe in EB individuals. The exception was for the older EB subject, who was the only one without an EB-type confirmatory diagnosis.

Considering that sugar consumption, tooth brushing frequency, and saliva parameters were similar when cases and controls were compared, a plausible explanation for a higher caries score would be the difficulties that the EB subjects have to perform their own tooth brushing. In addition, the bleeding and blisters can occur during oral hygiene in association to their limited mouth opening. In this regard, the maximum value for opening mouth recorded for an individual with $\mathrm{EB}(2.84 \mathrm{~cm})$ was far from the minimum one obtained by the individuals without $\mathrm{EB}$ $(4.3 \mathrm{~cm})$. Similar mouth opening limitation was described for patients with recessive dystrophic EB, who showed a mean value of $2.43 \mathrm{~cm} .{ }^{8}$ This limitation makes the whole cleaning procedure more difficult. In that way, for EB individuals, the physical condition - limited mouth opening and contracture of the fingers - could be added as etiological factors for dental caries to the classical ones already described. ${ }^{20,21}$

Finally, EB is considered as a rare disease, ${ }^{4,5,22}$ and because of that, there is a lack of information derived from clinical trials on oral health performed in a large group of subjects. Therefore, although the sample size of the present investigation is a limitation of this study, we believe that the outcomes obtained herein can contribute to the current knowledge about the influence of EB on the oral health status of individuals who present the disease. 


\section{CONCLUSION AND CLINICAL RELEVANCE}

To sum up, EB subjects presented higher caries scores than unaffected subjects and this fact might be associated with their physical condition. Salivary status was not different compared with health subjects. Due to the lack of oral clinical data related to the oral status of the disease, our findings add useful information regarding the relationship between caries prevalence and associated risk factors in EB subjects.

\section{ACKNOWLEDGMENT}

The authors would like to thank the great contribution of Prof Eduardo Barbosa Coelho for the statistical analysis.

\section{REFERENCES}

1. El Hachem M, Zambruno G, Bourdon-Lanoy E, Ciasulli A, Buisson C, Hadj-Rabia S, Diociaiuti A, Gouveja CF, Hernandez-Martin A, de Lucas Laguna R, et al. Multicentre consensus recommendations for skin care in inherited epidermolysis bullosa. Orphanet J Rare Dis 2014 May 20;9:76.

2. Salas-Alanis JC, Cepeda-Valdes R, Mellerio JE, Christiano AM, Uitto J. Progress in epidermolysis bullosa: summary of a workshop in CILAD-2010. Int J Dermatol 2012 Jun;51(6): 682-687.

3. Fine JD, Eady RA, Bauer EA, Bauer JW, Bruckner-Tuderman L, Heagerty A, Hintner H, Hovnanian A, Jonkman MF, Leigh I, et al. The classification of inherited epidermolysis bullosa (EB): report of the third International Consensus Meeting on Diagnosis and Classification of EB. J Am Acad Dermatol 2008 Jun;58(6):931-950.

4. Horn HM, Priestly GC, Eady RA, Tidman MJ. The prevalence of epidermolysis bullosa in Scotland. Br J Dermatol 1997 Apr;136(4):560-564.

5. Pope E, Lara-Corrales I, Mellerio J, Martinez A, Schultz G, Burrell R, Goodman L, Coutts P, Wagner J, Allen U et al. A consensus approach to wound care in epidermolysis bullosa. J Am Acad Dermatol 2012 Nov;67(5):904-917.

6. Harris CJ, Lucas VS, Bryan RA, Roberts GJ. Dental disease and caries related microflora in children with dystrophic epidermolysis bullosa. Pediatr Dent 2001 Sep-Oct;23(5): 438-443.
7. Primo BT, Costa DJ, Stringhini DJ, Rebellato NL, Moraes RS, Müller PR, Carneiro VL. Sialolithiasis in the duct of submandibular gland: a case report in patient with epidermolysis bullosa. J Contemp Dent Pract 2013 Mar 1;14(2):339-344.

8. Stellingsma C, Djkstra PU, Djkstra J, Duipmans JC, Jonkman MF, Dekker R. Restrictions in oral functions caused by oral manifestations of epidermolysis bullosa. Eur J Dermatol 2011 May-Jun;21(3):405-409.

9. Wright JT, Fine JD, Johnson LB. Oral soft tissues in hereditary epidermolysis bullosa. Oral Surg Oral Med Oral Pathol 1991 Apr;71(4):440-446.

10. Wright JT, Johnson LB, Fine JD. Developmental defects of enamel in humans with hereditary epidermolysis bullosa. Arch Oral Biol 1993 Nov;38(11):945-955.

11. Cagirankaya LB, Hatipoglu MG, Katipoglu H. Localized epidermolysis bullosa patient simplex with generalized enamel hypoplasia in a child. Pedatr Dermatol 2006 Mar-Apr; 23(2):167-168.

12. Dawes C. Salivary flow patterns and the health of hard and soft oral tissues. J Am Dent Assoc 2008 May;139 Suppl: 18S-24S.

13. World Health Organization. Oral health surveys: basic methods. 4th ed. Geneva: World Health Organization; 1997.

14. Gopinath VK, Arzreanne AR. Saliva as a diagnostic tool for assessment of dental caries. Arch Orofac Sci 2006;1: 57-59.

15. Leal SC, Bittar J, Portugal A, Falcão DP, Faber J, Zanotta P. Medication in elderly people: its influence on salivary pattern, signs and symptoms of dry mouth. Gerodontology 2010 Jun;27(2):129-133.

16. Nederfors T. Xerostomia and hyposalivation. Adv Dent Res 2000 Dec;14:48-56.

17. de Almeida Pdel V, Gregio AM, Machado MA, de Lima A, Azevedo LR. Saliva composition and functions: a comprehensive review. J Contemp Dent Pract 2008 Mar 1;9(3):72-80.

18. Edgar WM, Higham SM, Manning RH. Saliva stimulation and caries prevention. Adv Dent Res 1994 Jul;8(2):239-245.

19. Wright JT, Childers NK, Evans KL, Johnson LB, Fine JD. Salivary function of persons with hereditary epidermolysis bullosa. Oral Surg Oral Med Oral Pathol 1991 May;71(5):553-559.

20. Keys PH. Recent advances in dental research: bacteriology. Int Dent J 1962;12:443-464.

21. Intong LR, Murrell DF. Inherited epidermolysis bullosa: new diagnostic criteria and classification. Clin Dermatol 2012 Jan-Feb;30(1):70-77. 